\title{
ARTICLE
}

Received 8 Oct 2012 | Accepted 26 Feb 2013 | Published 16 Apr $2013 \quad$ DOl: 10.1038/ncomms2650

\section{Nuclear retention of the transcription factor NLP7 orchestrates the early response to nitrate in plants}

\author{
Chloé Marchive ${ }^{1,2}$, François Roudier ${ }^{3}$, Loren Castaings ${ }^{1,2, \dagger}$, Virginie Bréhaut $^{1,2}$, Eddy Blondet ${ }^{4}$,
} Vincent Colot $^{3}$, Christian Meyer ${ }^{1,2, \star} \&$ Anne Krapp ${ }^{1,2, \star}$

Nitrate is both an important nutrient and a signalling molecule for plants. Although several components of the nitrate signalling pathway have been identified, their hierarchical organization remains unclear. Here we show that the localization of NLP7, a member of the RWP-RK transcription factor family, is regulated by nitrate via a nuclear retention mechanism. Genome-wide analyses revealed that NLP7 binds and modulates a majority of known nitrate signalling and assimilation genes. Our findings indicate that plants, like fungi and mammals, rely on similar nuclear retention mechanisms to instantaneously respond to the availability of key nutrients.

\footnotetext{
${ }^{1}$ Institut National de la Recherche Agronomique (INRA), UMR1318, Institut Jean-Pierre Bourgin, Saclay Plant Sciences, RD10, F-78000 Versailles, France. ${ }^{2}$ AgroParisTech, Institut Jean-Pierre Bourgin, RD10, F-78000 Versailles, France. ${ }^{3}$ Institut de Biologie de I'Ecole Normale Supérieure, Centre National de la Recherche Scientifique (CNRS), UMR8197, Institut National de la Santé et de la Recherche Médicale (INSERM) U1024, 75230 Paris, France. ${ }^{4}$ URGV, UMR 1165 Institut National de la Recherche Agronomique-CNRS, 91057 Evry cedex, France. †Present address: Department of Plant Developmental Biology, Max Planck Institute for Plant Breeding Research, 50829 Cologne, Germany. ${ }^{\star}$ These authors contributed equally to this work. Correspondence and requests for materials should be addressed to A.K. (email: anne.krapp@versailles.inra.fr)
} 
$\mathrm{N}$ itrogen $(\mathrm{N})$, which is an essential macronutrient for living organisms, enters the food chain mainly through the assimilation performed by higher plants. Plant growth and crop productivity often are limited by low $\mathrm{N}$ availability in the soil. Therefore, nitrate has an important role in plant growth and development ${ }^{1}$. In addition to its role as a major nutrient for plants, nitrate acts as an important signalling molecule for several developmental processes such as root growth or flowering ${ }^{2}$. Indeed, nitrate controls the expression of over a thousand genes 3,4 ; however, the molecular players involved in this signalling pathway are largely unknown ${ }^{5}$. Indeed, only a few transcription factors (ANR1, NLP7, LBD37/38/39, SPL9 (refs 6-9)) implicated in the regulation of gene expression by nitrate have been identified, and most of these factors are encoded by genes that are themselves regulated by nitrate at the transcriptional level. One exception is NIN-Like Protein7 (NLP7), a putative transcription factor that is expressed constitutively and shares homology with NIN (for Nodule INception), an essential protein for nodulation in legumes ${ }^{10}$. Molecular and physiological analyses suggest that NLP7 is involved in plant responses to nitrate ${ }^{7}$. Here we show that, on nitrate supply, NLP7 is retained inside the nucleus to coordinate an early response to the availability of this essential nutrient.

\section{Results}

Nitrate triggers the nuclear accumulation of NLP7. To investigate if NLP7 itself is regulated by nitrate supply, we expressed a fully functional pNLP7:NLP7-GFP fusion construct
(Supplementary Fig. S1) in the nlp7-1 Arabidopsis mutant and examined the subcellular localization of this NLP7 fusion in response to nitrate. Whereas NLP7-GFP was mostly localized in the nucleus in the presence of nitrate (Fig. 1a), it was detected only in the cytoplasm after N-starvation (Fig. 1b). Resupplying nitrate after $\mathrm{N}$-starvation led, within minutes, to the relocation of NLP7-GFP into the nucleus (Fig. 1c,d, Supplementary Fig. S2 and Supplementary Movie 1). This relocalization was promoter independent as it occurred regardless of whether NLP7-GFP expression was driven by the constitutive CaMV 35S promoter or the native NLP7 promoter (Supplementary Fig. S3). Furthermore, this nuclear accumulation was rapidly reversible when nitrate was withdrawn (Fig. 1e-h, Supplementary Fig. S3e-h). Finally, this rapid nuclear relocation was specific to nitrate and independent of the counter cations $\mathrm{K}^{+}$or $\mathrm{NH}_{4}^{+}$as other $\mathrm{N}$ sources did not trigger nuclear translocation of NLP7-GFP (Supplementary Figs. S4 and S5). Based on these observations, we conclude that nitrate itself triggers the rapid relocation of NLP7 into the nucleus.

NLP7 localization is regulated by nitrate-dependent nuclear retention. The presence of a predicted leucine-rich nuclear export signal within NLP7 (Supplementary Fig. S6) prompted us to test if the cytoplasmic localization of NLP7 during nitrate starvation could result from its nuclear export by the Exportin1 CRM1/XPO1 (refs 11,12). In N-starved NLP7-GFP seedlings that had been treated for $3 \mathrm{~h}$ with leptomycin $\mathrm{B}$ (LMB), an inhibitor of Exportin1 (ref. 13), NLP7-GFP exhibited partial nuclear accumulation (Fig. 1i, Supplementary Fig. S3i). This nuclear
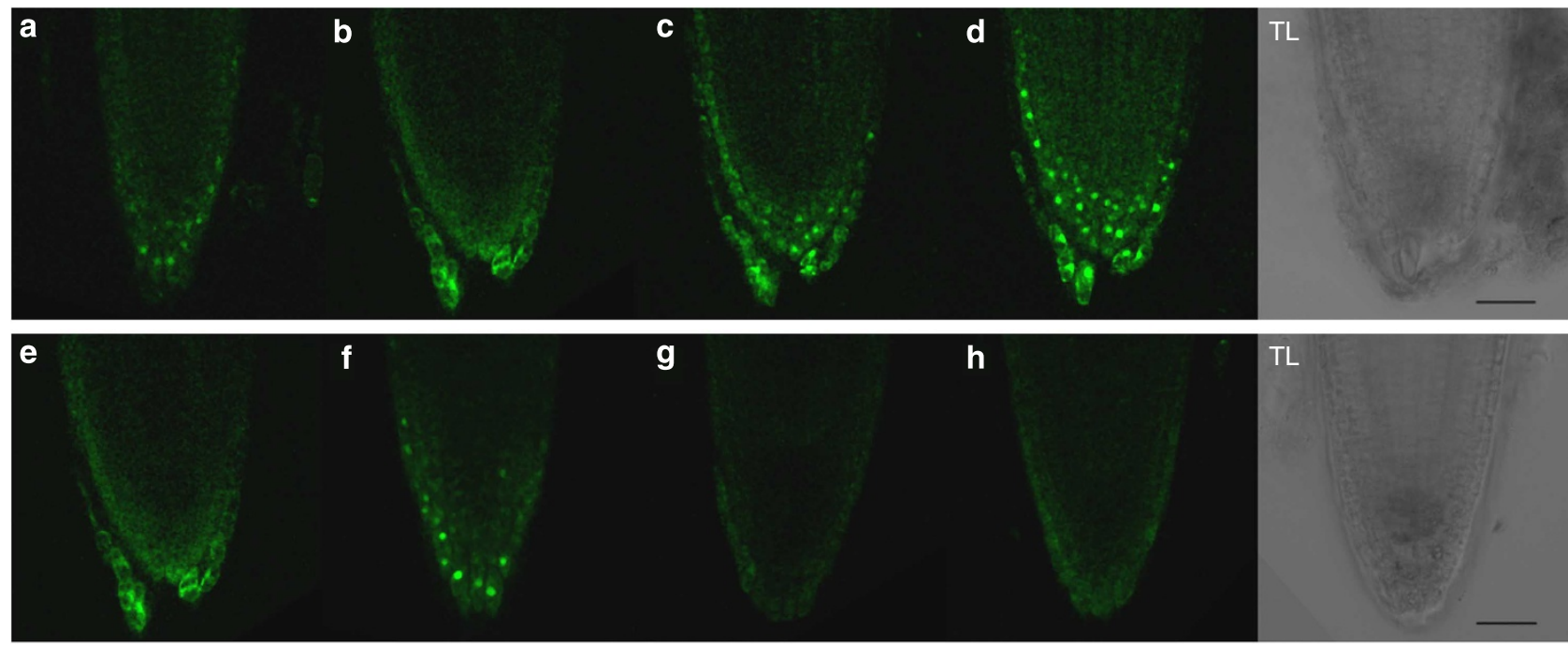

h

$\mathrm{TL}$
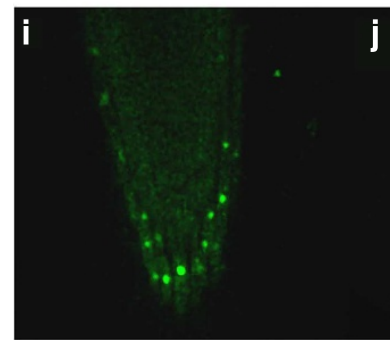

$\mathbf{k}$

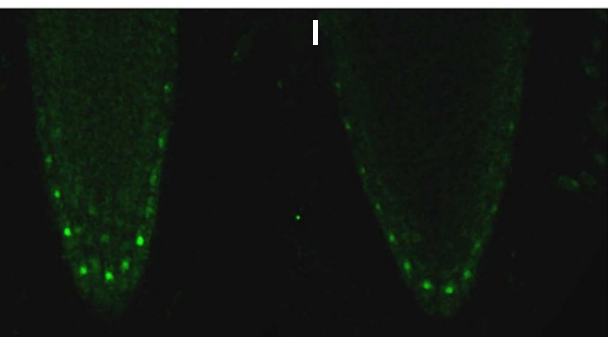

TL

Figure 1 | Nucleocytosolic shuttling of NLP7 is regulated by nitrate. Confocal imaging was performed on n/p7-1 seedlings expressing pNLP7-driven NLP7-GFP fusion protein. (a) Seedlings grown on nitrate. (b-d) Nitrate addition kinetics: N-starved seedlings (b) were transferred to a nitrate medium and observed after $3(\mathbf{c})$ and $6 \mathrm{~min}(\mathbf{d})$. (e-h) Nitrate depletion kinetics: $\mathrm{N}$-starved seedlings (e) were transferred to a nitrate medium for 20 min (f), then to $\mathrm{N}$-free medium and observed after $10(\mathbf{g})$ and $20 \mathrm{~min}(\mathbf{h})$. (i-I) Effect of leptomycin B (LMB) on the subcellular location of NLP7-GFP on nitrate addition and depletion. $\mathrm{N}$-starved seedlings were incubated with LMB in a $\mathrm{N}$-free medium for $3 \mathrm{~h}$ (i), then transferred for 20 min to nitrate medium (j) and thereafter depletion kinetics were recorded after 10 (k) or 20 min (I). Bright field images are shown for each time series (TL, transmitted light), the length of the scale bar corresponds to $50 \mu \mathrm{m}$. 
retention is consistent with the hypothesis that NLP7 is exported actively from the nucleus in the absence of nitrate rather than imported actively into the nucleus in the presence of nitrate. Also supporting active nuclear export, LMB treatment inhibited the rapid translocation of NLP7-GFP from the nucleus to the cytoplasm when nitrate-grown seedlings were shifted to $\mathrm{N}$-starvation (Fig. 1j-l, Supplementary Fig. S3j-l). Taken together, these data highlight the exquisite regulation of NLP7 subcellular location in response to nitrate availability.

NLP7 binds hundreds of genes including many genes involved in nitrate signalling and assimilation. Because NLP7 is a putative transcription factor, we sought to identify the genomic regions bound by NLP7 on its nitrate-induced relocation into the nucleus. Instead of using artificial, inducible nuclear localization methods, we took advantage of the rapid and specific nuclear relocalization of NLP7 that is induced by nitrate and subsequently identified NLP7-bound DNA regions. To this end, N-starved seedlings expressing pNLP7:NLP7-GFP in the nlp7-1 mutant background were resupplied with nitrate for $10 \mathrm{~min}$ before crosslinking and chromatin immunoprecipitation (ChIP) with an antiGFP antibody of the NLP7-bound chromatin fragments (Supplementary Fig. S7). By hybridizing the immunoprecipitated DNA to a whole-genome tiling array (ChIP-chip ${ }^{14}$ ), we identified 851 genes bound by NLP7 in the presence of nitrate (Supplementary Data 1). Gene Ontology (GO) term analysis revealed a significant enrichment for genes involved in N-metabolism and related metabolic pathways, such as the oxidative pentose-phosphate (OPP) pathway, sulphur- and carbon-metabolism, as well as genes involved in hormone metabolism and in transport (Fig. 2a). Moreover, among the bound genes were nearly all of those previously characterized as being involved in nitrate signalling, such as ANR1 (ref. 6), LBD37/38 (ref. 8), CIPK8 (ref. 15) and NRT1.1 (refs 16,17; Supplementary Table S1). As expected for a transcription factor, NLP7 binding was localized preferentially near the transcriptional start site of its target genes (Fig. 2b). Indeed, 511 out of the 851 gene sequences bound by NLP7 corresponded to 5 'promoter regions. However, no enriched DNA-binding motifs were found among the NLP7-immunoprecipitated sequences. For example, the NRE (nitrate response element) consensus sequence that was identified in the NiR promoter ${ }^{18}$ was not detected by pattern searching (RSAT tools ${ }^{19}$ ). Quantitative ChIP-PCR analysis was used to validate the NLP7 binding to four genes, NRT2.1, NRT2.2, NIA1 and LBD37, all of which are regulated by nitrate (Fig. 2c,d). In the case of NIA1, NLP7 binding was also detected downstream of the gene, consistent with the requirement of additional $3^{\prime}$ end sequences for the proper induction of this gene by nitrate ${ }^{20}$. To directly test the effect of NLP7 on its bound genes, transient transactivation assays were performed in Arabidopsis protoplasts using the reporter construct pNRT2.1:LUC, which contains the luciferase gene under the control of the NRT2.1 promoter $^{21}$ and includes the sequence bound by NLP7. Co-transformation of pNRT2.1:LUC with p35S:NLP7 increased the luciferase activity sevenfold compared with the control pNRT2.1:LUC without p35S:NLP7 (Fig. 2e). Taken together, these results show that NLP7 is a bona fide transcription factor that likely acts as a master regulator of the early response to nitrate.

NLP7 broadly impacts the rapid regulation of gene expression by nitrate. To further assess the role of NLP7 in the early response to nitrate, we profiled, genome wide, the transcripts of wild type (wt) and two allelic loss-of-function $n l p 7$ mutant plants during a short-term nitrate-resupply kinetics and compared their respective nitrate response transcriptomic profiles (Fig. 3a, Supplementary Data 2). We found that $25 \%$ of the genes in the wt that were differentially regulated after a 10 min nitrate supply following $\mathrm{N}$-starvation showed a weaker or an opposite response in at least one of the two $n l p 7$ mutants $\log _{(2)}$ expression ratios either $\geq 0.5$ or $\leq-0.5,176$ out of 712 genes, Supplementary Data 3). Moreover, after 10 and 20 min of nitrate induction, the fraction of genes that was deregulated in at least one of the $n l p 7$ mutants increased to 58\% (383 out of 661 genes, Supplementary Data 4). These results indicate that NLP7 has a profound and broad influence on the early transcriptome response to nitrate.

NLP7 binding leads to both activation and repression of nitrate-responsive genes. To clearly distinguish between direct and indirect effects, we determined whether the genes that showed a modified nitrate response in the $n l p 7$ mutants were bound by NLP7. In total, we identified 91 genes that both exhibited an altered expression in the $n l p 7$ mutants in response to the nitrate signal and were bound by NLP7. Among these direct targets of NLP7 were nearly proportionate numbers of genes that were either less induced or less repressed by nitrate in the $n l p 7$ mutants compared to wt (Supplementary Data 5). Whether NLP7 activates or represses a target gene presumably is determined by the structure of the promoter, the binding sites, the flanking sequences and the interplay with other transcriptional regulators as previously observed ${ }^{22,23}$. To deepen this analysis, we clustered the nitrate-responsive genes according to their expression pattern in wt plants (Fig. 3b, Supplementary Fig. S8 and Supplementary Data 6). In the highly nitrate-inducible cluster IV, 36 out of 79 genes were bound by NLP7, representing the highest proportion of bound genes among the clusters. Almost half of these 79 genes showed modified nitrate response kinetics in the $n l p 7$ mutants, with mainly a decreased induction compared with the wt, indicating that NLP7 is an activator of these nitrateinduced genes (Fig. 3b, Supplementary Data 7). For clusters V and VI, which correspond to nitrate induction between 0 and $10 \mathrm{~min}$ and between 10 and $20 \mathrm{~min}$, respectively, one-third of the NLP7-bound genes showed modified nitrate response kinetics in the $n l p 7$ mutants. Within the nitrate-repressed or transiently induced genes (clusters I, II and III) the majority of the few NLP7-bound genes $(<10 \%)$ in these clusters were upregulated in the $n l p 7$ mutants compared with the wt (Fig. 3b, Supplementary Data 7), suggesting that, in these cases, NLP7-dependent nitrate repression was impaired.

NLP7 directly regulates different steps of $\mathrm{N}$ assimilation. The most highly enriched GO terms among the direct NLP7 targets that both are detected by ChIP-chip and less nitrate-induced in $n l p 7$ mutants are $\mathrm{N}$ assimilation and the OPP pathway (Supplementary Fig. S9). The OPP pathway is a critical step providing reductants for nitrate assimilation and has been shown to have a very early response to nitrate resupply ${ }^{9}$. Accordingly, the list of NLP7-dependent nitrate-inducible genes includes nitrate transporter NRT2.1, nitrate reductase NIA1, nitrite reductase, glutamine synthetase 2, uroporphyrinogen III methyltransferase 1, ferredoxin 3 and transketolase (At3g60750) (Fig. 4a, Supplementary Fig. S10 and Supplementary Data 7). In addition, putative new regulatory proteins involved in the early nitrate signalling were identified within the NLP7 targets such as MAPKKK13 (MEKK13), bHLH34 and NLP3 (Fig. 4b, Supplementary Fig. S11 and Supplementary Data 7).

We confirmed the modified nitrate-responsive expression pattern of these genes by quantitative reverse transcription-PCR (Supplementary Figs S10 and S11). This highly sensitive detection method allowed us to show that several of the lowly expressed genes bound by NLP7, for which no differential expression between $n l p 7$ mutants and wt was detected in our microarray 
a

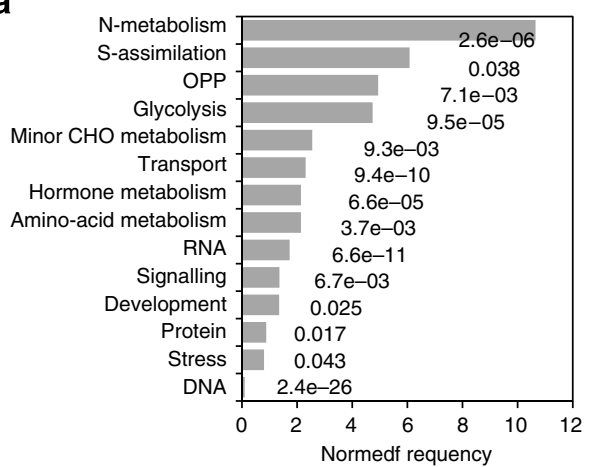

b

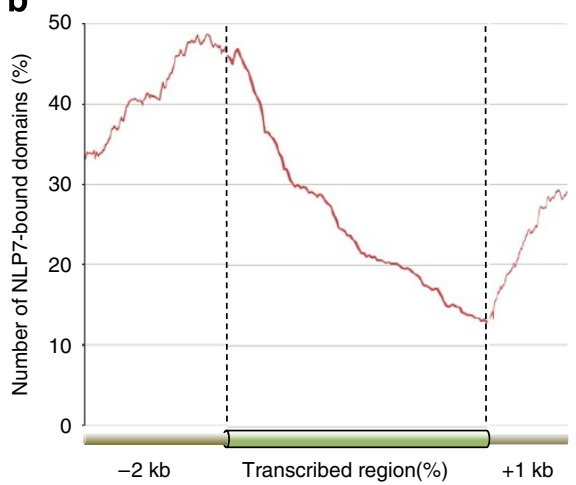

C
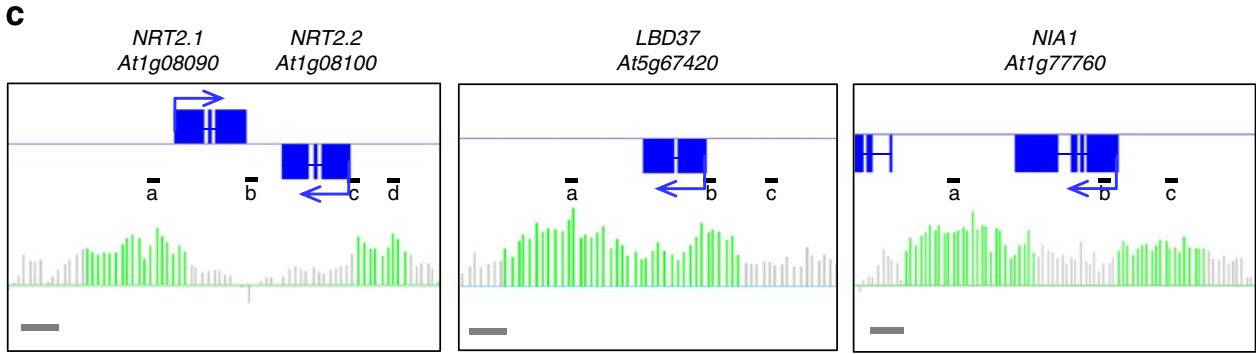

d
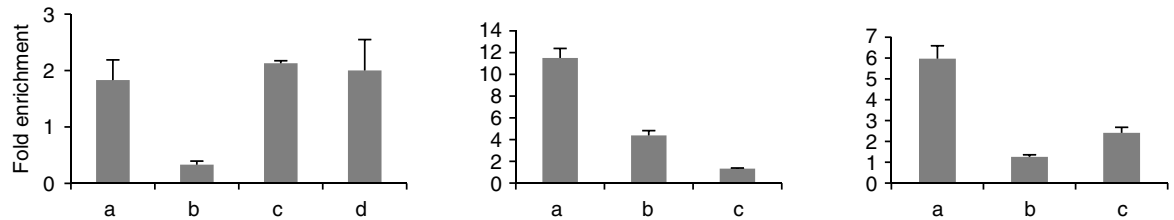

e

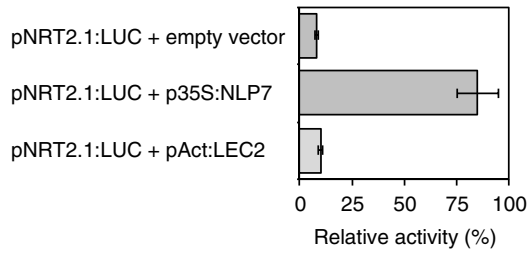

Figure 2 | Genome-wide NLP7 binding in response to nitrate. (a) Gene ontology (GO) term enrichment for the high confidence NLP7 target genes. GO terms were defined using Mapman bins and sorted on frequency related to Arabidopsis set. Associated $P$-values of the hypergeometric distribution are indicated, which were calculated as follows: $P=B C(M, x) \times B C(N-M, n-x) / B C(N, n)$. BC is the binomial coefficient, calculated as follows: $\mathrm{BC}(n, k)=n ! /(k ! \times[n-k] !)$. In the above equations, $x$ is the number of input genes with the selected classification, $n$ is the total number of input genes, $M$ is the number of genes with the selected classification in the database (MapMan) and $N$ is the total number of genes in this database. (b) Spatial distribution and number of NLP7-bound domains in proximity to transcribed regions. To account for different gene size, the positions of binding sites within transcribed regions were calculated relative to their length. (c,d) ChIP-chip and ChIP-qPCR data at selected NLP7 target genes. (c) The exon-intron structure and orientation of target genes are shown in the top half of each panel. NLP7 ChIP-chip profile is shown at the bottom half; probes reporting a significant enrichment of NLP7 binding in response to nitrate are indicated in green. Amplicons analysed in $\mathbf{d}$ are given as black line and numbered a-d. Scale bar, 1 kb. (d) Real-time ChIP-PCR at selected targets on an independent biological replicate. Position of amplified fragments is indicated in c. Each PCR amplification of the immunoprecipitated samples was normalized to the respective input. The fold enrichment of the nitrate-induced NLP7 binding was calculated as the ratio between the + nitrate and the $-\mathrm{N}$ immunoprecipitated amplification signals. (e) Transactivation of pNRT2.1:LUC by NLP7. After co-transfection of pNRT2.1:LUC, p35S:NLP7 and p35S:GUS in Arabidopsis protoplasts, luciferase activity was measured relative to p35S:GUS activity. pACT:LEC2 was used as a control transcription factor.

analyses, were indeed differentially regulated by nitrate in the $n l p 7$ mutant background. This was the case for the LBD37, LBD38, MAPKKK14 (MEKK14) and HYH genes (Supplementary Fig. S11).

NLP7 binds to nitrate-responsive genes. A large fraction of the NLP7-bound genes was not differentially expressed in the $n l p 7$ mutants compared to the wt during the early response to nitrate. This result is not surprising as binding of a given transcription factor, as detected by ChIP-chip, does not necessarily correlate with effective transcription modulation of the corresponding gene by the same factor ${ }^{24}$. The action of transcription factors is modulated by the genomic landscape of DNA regulatory sequences and co-regulatory proteins. In addition, some of the NLP7-bound genes have very low expression levels, and, thus, the microarray analysis might have failed to determine their expression differences, such as was the case for $L B D 37, L B D 38$, MAPKKK14 (MEKK14) and HYH. In addition, some of the 
a

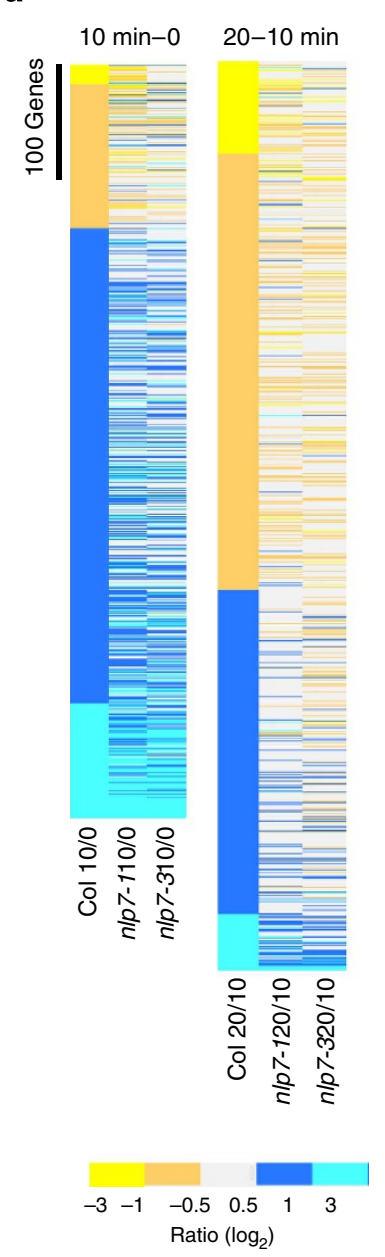

b
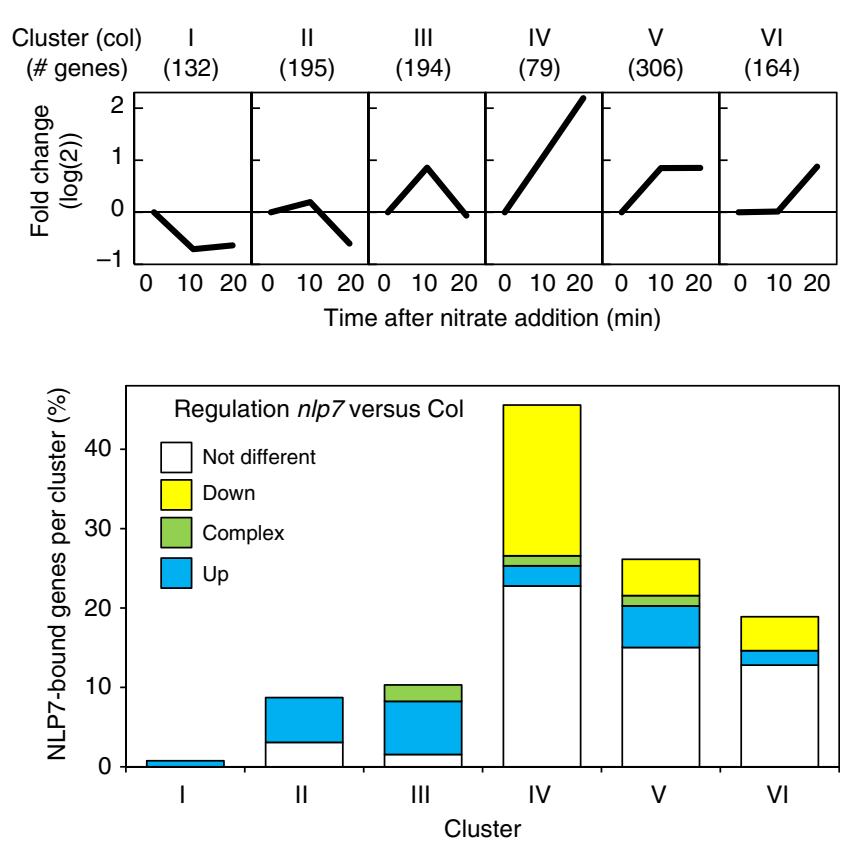

c

NLP7-bound

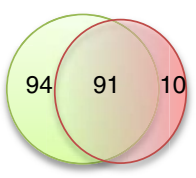

Nitrate-regulated NLP7-regulated

Figure 3 | NLP7-binding and direct regulation of nitrate-regulated gene clusters. (a) Differential expression of genes in nitrate-induced wt (Col) and nlp7 mutants. N-starved seedlings were exposed to nitrate for 10 or 20 min and transcriptome variations were analysed using CATMA arrays. Differentially expressed genes $\left(\log _{(2)}\right.$ expression ratios either $>0.5$ or $\left.<-0.5\right)$ were ordered from the lowest to the highest ratio using the wt (Col) comparisons as reference. The corresponding ratios in nitrate response of $n l p 7$ mutants are shown for each gene (0, 10, 20: time in minutes after nitrate supply).

(b) Distribution of NLP7-bound genes within nitrate-regulated gene clusters. Top panel, six gene clusters (I-VI) were defined based on the expression pattern observed in the wt after nitrate supply. The numbers of genes in each cluster as well as their average expression trend (black line) are given. Bottom panel, distribution of NLP7-bound genes in the different clusters according to their regulation by nitrate in the nlp7 mutants in comparison to the wt: down (yellow), up (blue), not different (white). Complex (green) stands for a mixed up/down pattern at the two time points (10 and 20 min). (c) Venn diagram showing the number of NLP7-bound genes that are differentially expressed in nlp7 mutants (red circle) and NLP7-bound genes that are regulated by nitrate (green circle).

NLP7 targets may require longer periods of nitrate induction to show significant expression changes. It is also possible that some targets may be regulated by NLP7 only after accumulation of a cofactor not present in our experimental condition or may be expressed in specific cells or developmental stages excluded from our study. Indeed, in addition to the 91 NLP7-bound genes that are deregulated for early nitrate response in the $n l p 7$ mutants, there are 94 NLP7-bound genes that are regulated by nitrate during our short nitrate supply kinetic, but do not show a differential response in the $n l p 7$ mutant (Fig. 3c, Supplementary Data 8). For these genes, deregulated expression in the mutants might have been missed in our experimental conditions. In total, the percentage of nitrate-regulated genes among the NLP7bound genes is four times higher than the genome-wide $3.8 \%$ of nitrate-regulated genes in our short-term nitrate supply experiment. It is important to note that among the NLP7bound and differentially expressed genes in the $n l p 7$ mutants, only a small fraction (10 out of 101) corresponds to genes that are not regulated by nitrate (Fig. 3c, Supplementary Table S2), pointing clearly to the fact that NLP7 is primarily implicated in nitrate signalling.

\section{Discussion}

We showed here that the transcription factor NLP7 is a main regulator of nitrate signalling. The intracellular localization of NLP7 is specifically and rapidly regulated by nitrate and mediated by a nuclear retention mechanism. In plants, nucleocytosolic shuttling is a major regulatory mechanism involved, for example, in the signalling cascades triggered by light, by brassinosteroids or plant pathogen interaction ${ }^{25}$. However, no such mechanism has been identified for nutrient signalling in plants so far. Our data indicate that the nuclear export of NLP7 slows down within minutes after the addition of nitrate, allowing a rapid NLP7dependent regulation of gene expression. Consistent with our data, Krouk et al. ${ }^{9}$ showed that gene expression is regulated by nitrate as rapidly as 2 min after supply. 

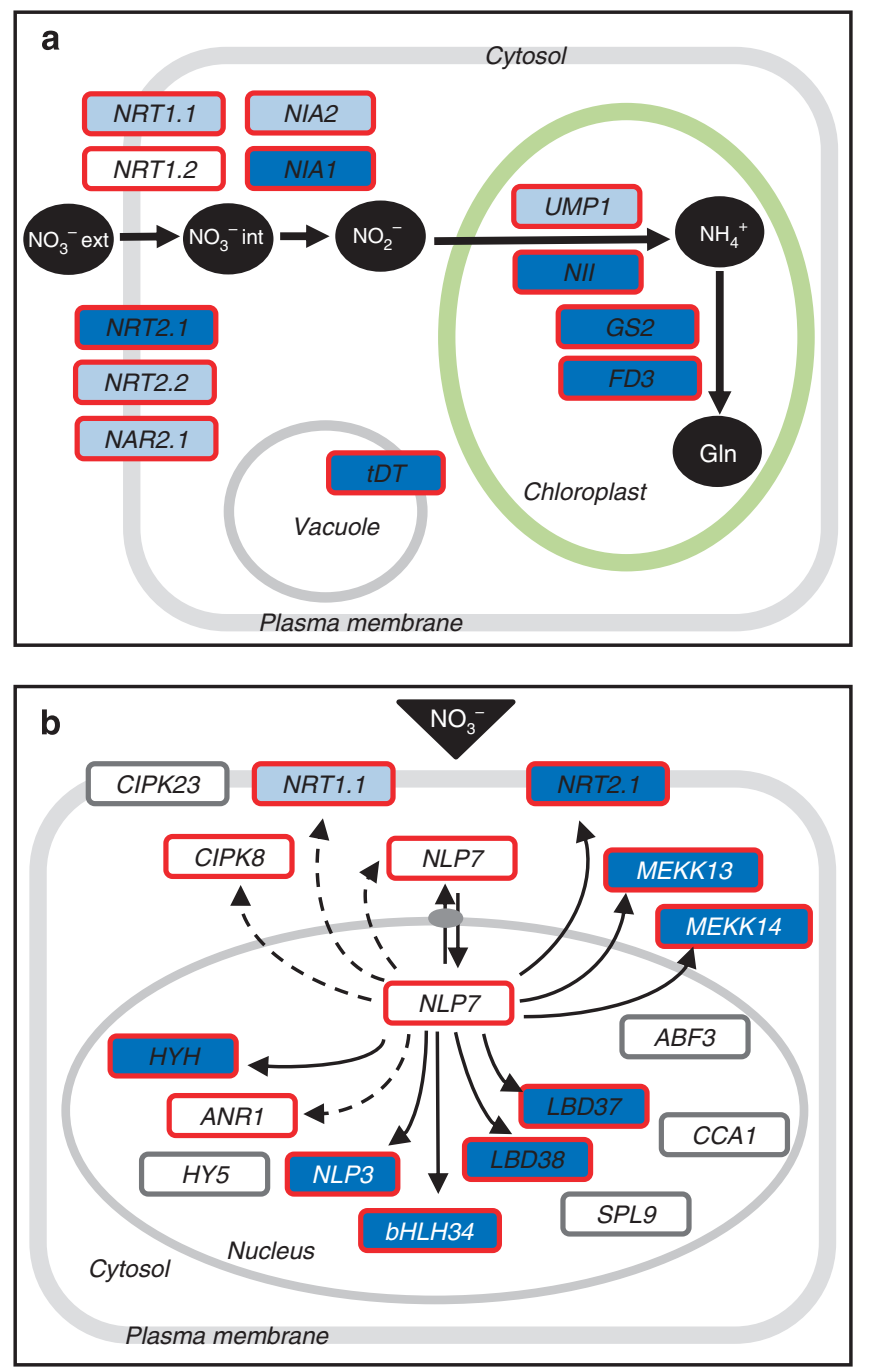

Figure 4 | NLP7 directly regulates many steps of the primary N assimilation and nitrate signalling pathways. (a) $\mathrm{N}$ assimilation pathway from nitrate uptake on the plasma membrane to ammonium incorporation into C-skeletons. Depicted is a plant cell, with plasma membrane, cytosol, chloroplast and vacuole. NRT, nitrate transporter; NAR2, nitrateassimilation-related 2 (NRT3.1); NIA, nitrate reductase; NII, nitrite reductase; UMP1, S-adenosyl-L-methionine-dependent uroporphyrinogen III methyltransferase 1; GS2, glutamine synthetase; GOGAT, glutamineoxoglutarate-aminotransferase (GLU1, GLU2, GLS); tTD, tonoplastic malate/fumarate transporter. (b) Nitrate signalling from nitrate perception by nitrate transceptors NRT1.1 and NRT2.1 to the regulation of gene expression. All known and several putative regulators (black and white writing, respectively) are depicted in a plant cell with plasma membrane, cytosol and nucleus. NRT, nitrate transporter/nitrate sensor; CIPK, calcineurin B-like (CBL)-interacting protein kinase; MEKK, mitogenactivated kinase kinase kinase; $H Y 5$, long hypocotyl5; HYH, HY5 homologue; ANR1, Arabidopsis nitrate regulated 1; $b H L H$, basic helixloop-helix transcription factor; $L B D$, lateral organ boundary domain; $A B F 3$, auxin-binding factor 3; CCA1, circadian clock associated 1; SPL9, squamosa promoter binding protein-like 9 . The colour of the border of each gene represents the results from the ChIP-chip experiment: genes with a red border are bound by NLP7, genes with a grey border are not bound by NLP7. Dark and light blue represent the results from expression profiling during nitrate addition kinetics; light blue: gene was induced by nitrate, dark blue: gene was induced by nitrate and less nitrate-regulated in the n/p7 mutants. Black letters: genes already characterized, white letters, newly identified gene as putatively involved in nitrate signalling. All gene products might not occur in the same cell type.
Our work identifies direct NLP7 target genes. Through ChIPchip we identified 851 NLP7-bound genes only after a 10 minresponse to nitrate. Consistent with the role of NLP7 in nitrate response, NLP7-bound genes are highly enriched for regulatory and structural genes of nitrate metabolism and signalling. NLP7bound domains possess an average size of $1,114 \mathrm{bp}$ (with a mean size of $859 \mathrm{bp}$ ). However, pattern searching did not detect any significant consensus motifs among the NLP7-bound fragments. Previous searches for nitrate-responsive elements have been conducted, but, with the exception of the NRE, which is involved in nitrate regulation of $\mathrm{NiR}$ (ref. 18), no such elements have been identified so far. We did not find enrichment of the NRE sequence among the NLP7-bound fragments. Moreover, Chlamydomonas NIT2, a RWP-RK protein related to NLP7, also has been shown to bind sequences unrelated to NRE elements $^{26}$, indicating that the RWP-RK proteins are not likely candidates for NRE-binding proteins.

Our ChIP-chip analysis together with our genome-wide analysis of nitrate-regulated gene expression in $n l p 7$ mutants under the same experimental conditions, allowed us to identify direct target genes of NLP7. We showed that the loss of NLP7 leads to a sizeable rearrangement of the transcriptional response to nitrate, impacting the expression of genes involved in both nitrate signalling and assimilation. Moreover, NLP7 was shown to act as both a transcriptional activator and a repressor depending possibly on its regulatory partners. Our rather stringent approach likely underestimated the number of genes that are regulated directly by NLP7 in response to nitrate due to, on the one hand, the low expression of putative target genes, and on the other hand, to possible redundant regulation by other transcription factors, such as other NLPs. Indeed, the sequence of NLP6 is highly homologous to NLP7. Thus, it is possible that NLP6 and other NLPs could bind some NLP7 targets and regulate their expression in the absence of NLP7. However, the fact that the nlp7 mutants, but not other nlp mutants, have a clear nitraterelated phenotype ${ }^{7}$ supports the hypothesis that NLP7 is the major regulatory element among NLP proteins.

Together, the overlap between NLP7-bound genes and deregulated gene expression in $n l p 7$ mutants highlighted a subset of genes mainly related to nitrate assimilation and oxidative pentose pathway, suggesting that NLP7 is one of the master regulators of nitrate signalling.

However, a significant fraction of the nitrate-regulated genes were not bound by NLP7. These genes may either be regulated by other signalling pathways or be indirectly controlled by NLP7 through NLP7-dependent regulators. Indeed, the fact that NLP7 loss-offunction does not totally abolish the responses to nitrate (Supplementary Fig. S1) indicates that NLP7-independent nitrate signalling cascades likely exist. Consistent with this observation, four other NLP genes have been identified as direct targets of NLP7 (NLP1, NLP2, NLP3 and NLP5; Supplementary Table S1). Our data indicates that the hypothesis that tight regulation between NLP proteins controls nitrate signalling needs to be challenged.

The deregulation of direct NLP7 targets has far-reaching consequences for genome-wide nitrate regulation. Indeed, the transcriptomic changes that result from NLP7 loss-of-function extend beyond the genes directly bound by NLP7. Secondary effects arising from the loss-of-function of NLP7 are larger (Supplementary Fig. S8) than changes of transcript abundance for NLP7-bound genes. These indirect target genes might be under the control of additional regulatory circuits, as regulatory proteinencoding genes are themselves bound and transcriptionally regulated by NLP7. Thus, a small deregulation of a subset of genes targeted by NLP7 can have an expansive effect on nitrate-responsive genome-wide gene expression, pinpointing the upper hierarchical role of NLP7 as an orchestrator of nitrate responses. 
Taken together, the swift regulation of NLP7 by nitrate and the functional diversity of NLP7 target genes highlight the important and broad role of NLP7 in the plant primary nitrate response. Indeed, NLP7 regulates the expression of genes involved in a range of biological activities from nitrate transport and assimilation to transduction of the nitrate signal by various regulators. This coordinated regulation by NLP7 ensures rapid adaptation to $\mathrm{N}$ availability to maintain plant nitrate homeostasis. Unlike most signalling molecules, nitrate is also a main nutrient for plants. In Saccharomyces species, nutrients have been shown to regulate the nuclear export of tRNAs ${ }^{27,28}$. Plants, like mammals, do not seem to directly regulate nuclear export of tRNA in response to nutrient stress ${ }^{29}$. So far, nutrient-dependent regulation of the nuclear export of transcription factors has been shown for NirA, the master regulator of $\mathrm{N}$ assimilation in the filamentous fungus Aspergillus ${ }^{30}$, and for FoxO1, a Forkhead box transcription factor involved in the regulation of metabolism in mammals ${ }^{31}$. Therefore, our finding that nuclear export of NLP7 is regulated by nitrate suggests convergent evolution in distant organisms of mechanisms that enable instant responses to the availability of key nutrients.

\section{Methods}

Plant material and culture conditions. The two $n l p 7$ allelic knockout mutants, $n l p 7-1$ and $n l p 7-3$, were in the Columbia (Col-8) wt background of Arabidopsis thaliana. The nlp7-1 mutant background was used for complementation by the pNLP7:NLP7-GFP or by $\mathrm{p} 35 \mathrm{~S}:$ NLP7-GFP constructs ${ }^{7}$. Plants were grown on a $3 \mathrm{mM} \mathrm{KNO}_{3}$ liquid medium $\left(3 \mathrm{mM} \mathrm{KNO}_{3}, 1 \mathrm{mM} \mathrm{KH_{2 }} \mathrm{PO}_{4} / \mathrm{K}_{2} \mathrm{HPO}_{4}, 0.5 \mathrm{mM}\right.$ $\mathrm{CaSO}_{4}, 0.5 \mathrm{mM} \mathrm{MgCl}_{2}, 3 \mathrm{mM}$ MES, pH 5.8, 0.25\% (w/v) sucrose, supplemented with microelements (modified from (ref. 4)) in long-day photoperiod $\left(16 / 8 \mathrm{~h}\right.$ at $25^{\circ} \mathrm{C}$ with $80 \mu \mathrm{mol}$ photons. $\left.\mathrm{m}^{-2} \mathrm{~s}^{-1}\right)$.

Confocal imaging. Plants were grown for 5 days on the $3 \mathrm{mM} \mathrm{KNO}_{3}$ liquid medium (see above), nitrogen-starved for 2 days by exchanging the culture medium with a nitrogen-free medium, in which $\mathrm{KNO}_{3}$ was replaced by $\mathrm{KCl}$. Plants were mounted in $40 \mu \mathrm{l}$ of a top agar $(0.1 \%$, w/v) solution. During confocal imaging, either $5 \mu \mathrm{l}$ of $0.5 \mathrm{M} \mathrm{KNO}_{3}, 5 \mu \mathrm{l}$ of $0.5 \mathrm{M} \mathrm{NH} \mathrm{NH}_{4} \mathrm{Cl}, 2.5 \mu \mathrm{l}$ of $0.5 \mathrm{M} \mathrm{NH}_{4} \mathrm{NO}_{3}, 15 \mu \mathrm{l}$ of $50 \mathrm{mM}$ glutamine or $5 \mu \mathrm{l}$ of $0.5 \mathrm{M} \mathrm{KNO}_{2}$ were added to the mounting medium; the same plantlets were imaged during a time course. For nitrate depletion, plantlets were grown as described above, then transferred for $20 \mathrm{~min}$ to $3 \mathrm{mM}$ nitrate medium, and finally transferred to $\mathrm{N}$-free medium. At each time point of the kinetics, a new plantlet was withdrawn from the medium for immediate imaging. For the nuclear export inhibition experiment, a 3-h preincubation with LMB (Sigma, at $100 \mathrm{nM}$ final concentration) was performed in the $\mathrm{N}$-free medium. LMB was then added at each step.

Laser scanning confocal imaging was performed using the Zeiss 710 microscope equipped with an argon laser (488 $\mathrm{nm}$ for green fluorescent protein (GFP) excitation). Emission was collected at 510 to $545 \mathrm{~nm}$ (GFP). The images were coded green for GFP. For nuclei staining, plantlets were stained with 4', 6-diamidino-2phenylindole (DAPI, $5 \mu \mathrm{g} \mathrm{ml}^{-1}$; Invitrogen). The excitation wavelength was $405 \mathrm{~nm}$ and the emission was collected at $420-480 \mathrm{~nm}$. A sequential scanning mode was used when DAPI marker staining was combined with GFP to minimize the crosstalk between the two partially overlapping emission spectra. The images were coded green (GFP) and red (DAPI) giving yellow co-localization in merged images. Images were processed in ImageJ and Photoshop Element (Adobe Systems).

Chromatin immunoprecipitation. Seedlings were grown for 10 days on a $3 \mathrm{mM}$ $\mathrm{KNO}_{3}$ liquid medium. Plants were then nitrogen-starved for 3 days by changing the culture medium with a nitrogen-free medium, in which $\mathrm{KNO}_{3}$ was replaced by $\mathrm{KCl}$. A 10-min nitrate induction was performed by changing the culture medium for the $3 \mathrm{mM}$ nitrate solution $(+\mathrm{N})$. For the control samples $(-\mathrm{N})$, the nitrogen-free solution was changed for a fresh one.

ChIP was performed essentially, as described in Gendrel et al., ${ }^{32}$ using $3.5 \mu \mathrm{l}$ of anti-GFP antibody and Dynabeads coupled with Protein A and G (Invitrogen). Reverse crosslinking was carried out with the iPure kit (Diagenode). Immunoprecipitated DNA was amplified using the Whole Genome Amplification 4 kit (Sigma), differentially labelled and co-hybridized in dye-swap experiments according to the manufacturer's instructions (Roche NimbleGen). ChIP-chip analysis of NLP7 binding regions was performed in two biological replicates (two-dye swaps).

ChIP-chip analysis. Hybridization data were normalized using an analysis of variance model to remove technical biases. Data were averaged on the dye-swap to remove tile-specific dye biases and across biological replicates. Normalized data were then analysed using a three-states Parsimonious Higher-Order Hidden Markov Model (PHHMM, www.jstacs.de/index.php/PHHMM) to model the joint distribution of the $+\mathrm{N}$ and $-\mathrm{N}$-derived signals, taking into account spatial dependency between the DNA sequence reported by the probes on the tiling array $^{33}$. Briefly, normalized log $2+\mathrm{N} /-\mathrm{N}$ signal ratio for each probe corresponds to the observed values of the Hidden Markov chain, while the hidden chain is the enrichment state of the DNA sequence reported by each probe in the ChIP-chip experiments, respectively. This PHHMM procedure was applied to the data following a two-step process. First, initial model parameters are iteratively maximized by a specific Bayesian Baum-Welch training algorithm enabling the integration of previous knowledge and by a data-dependent reduction of transition parameters. Second, based on this training, optimized model parameters are obtained that allow the parsimonious higher-order HMM with univariate Gaussian emission distributions to effectively model spatial dependencies between measurements ${ }^{33}$. This HMM approach provides a probabilistic classification of each probe into one of the three states, namely non-enriched, enriched and depleted in the $+\mathrm{N} /-\mathrm{N}$ comparison. Adjacent probes belonging to the same HMM-derived state are joined into domains by requiring a minimal run of $400 \mathrm{bp}$ and allowing a maximal gap of $200 \mathrm{bp}$. An enrichment probability is assigned to each domain by calculating the median probability of each probe within a domain. Data were deposited in Gene Expression Omnibus (GEO) under the accession number GSE36763.

ChIP-PCR. The ChIP-chip data were validated by ChIP-quantitative PCR (qPCR), on the two hybridized biological replicates as well as on a third biological replicate. qPCR was performed using LightCycler-FastStart DNA Master SYBR Green I kit (Roche) according to the manufacturer's protocol on a Eppendorf Realplex qPCR machine. Specific primer sets are given in Supplementary Table S3. Each amplification of the immunoprecipitated samples was normalized to the respective input. The fold enrichment of the nitrate-induced NLP7 binding was calculated as the ratio between $+\mathrm{N}$ and the $-\mathrm{N}$ immunoprecipitated amplification signal.

Transcriptome analysis. Plantlets (Col-8, $n l p 7-1$ and $n l p 7-3$ mutants) were grown as described for ChIP. The whole plantlets were collected at time point 0 (before nitrate addition), 10 and 20 min after nitrate addition. Total RNA extractions from two independent biological replicates were performed using GenElute Mammalian Total RNA kit from Sigma-Aldrich, modified by adding a DNase step (Qiagen RNase-free DNase kit).

Microarray analysis was carried out at the Research Unit in Plant Genomics in Evry, France, using the CATMA array (V5) containing 24,576 gene-specific tags corresponding to 22,089 genes from Arabidopsis ${ }^{34}$. The analysis was performed as in Lurin et al. ${ }^{35}$ In short, cRNAs were produced from $2 \mu \mathrm{g}$ of total RNA (Message Amp aRNA kit, Ambion). Then $5 \mu \mathrm{g}$ of cRNAs were reverse transcribed in the presence of 300 units of SuperScript II (Invitrogen), cy3-dUTP, and cy5-dUTP (NEN), and purified and concentrated with YM30 Microcon columns (Millipore). Slides were prehybridized for $1 \mathrm{~h}$ and hybridized overnight at $42{ }^{\circ} \mathrm{C}$ in $25 \%$ formamide. After washing the arrays were scanned, and images were analysed by GenePix Pro 3.0 (Axon Instruments). For each comparison one technical replication with fluorochrome reversal was performed for each biological duplicate. For the wt (Col-8) transcriptome analysis, the following sample comparisons were performed in two biological replicates: $\mathrm{Col} 10 \mathrm{~min}$ vs Col T0; Col $20 \mathrm{~min}$ vs Col $10 \mathrm{~min}$. For each mutant the following sample comparisons were done: $n l p 7 \mathrm{~T} 0$ vs Col T0; $n l p 710 \mathrm{~min}$ vs Col $10 \mathrm{~min}$ and nlp7 $20 \mathrm{~min}$ vs Col $20 \mathrm{~min}$.

Analysis of transcriptome data. Experiments were designed with the Bioinformatic and Predictive Genomics group at the Unite de Recherche en Génomique Végétale in Evry, France. Specific statistics were developed to analyse CATMA hybridizations. For each array, the raw data comprise the logarithm of median feature pixel intensity (in log base 2) at wavelengths of $635 \mathrm{~nm}$ (red) and $532 \mathrm{~nm}$ (green). No background was subtracted. An array-by-array normalization was performed to remove systematic biases as described before ${ }^{35}$. In short, after excluding bad spots, a global intensity-dependent normalization using the Loess procedure was performed to correct the dye bias, followed by a correction for print tip effects on each metablock. To determine differentially expressed genes, we performed a paired $t$-test on the log ratios averaged on the dye swap. A trimmed variance was calculated from spots that did not display extreme variance. The raw $P$-values were adjusted by the Bonferroni method, which controls the family-wise error rate (with a type I error equal to $5 \%$ ). We also adjusted the raw $P$-values to control a false discovery rate using Benjamini-Yetkutieli at a level of $1 \%$. In the CATMA analysis pipeline, family-wise error rate proved to be the best solution to balance the estimated number of false positives and false negatives ${ }^{36}$. As described before $^{37}$, when the Bonferroni $P$-value was lower than 0.05 , the gene was declared differentially expressed. Microarray data were deposited in GEO under the accession number GSE39261.

GO terms were defined using the classification superviewer tool at http://bar.utoronto.ca/ntools/cgi-bin/ntools_classification_superviewer.cgi using Mapman bins and sorted on frequency related to Arabidopsis set. 
Quantitative reverse transcription-PCR. RNA was extracted with Trizol method (Invitrogen). First-strand cDNAs were synthesized using Moloney murine leukaemia virus reverse transcriptase and oligo $(\mathrm{dT})_{15}$ primers (Promega) ${ }^{38,39}$. PCR was performed as for ChIP-PCR. Specific primer sets are given in Supplementary Table S3.

Promoter transactivation in Arabidopsis protoplasts. For promoter transactivation assays, Arabidopsis protoplasts were prepared from cell cultures by a sucrose gradient after $3 \mathrm{~h}$ of cell wall digestion as in Thomine et al. ${ }^{39}$ polyethylene glycol (PEG)-mediated transfection was performed using $10 \mu \mathrm{g}$ of plasmid DNA. Plasmids were obtained from M. Lepetit (pNRT2.1:LUC) ${ }^{21}$ and from B. Dubreucq $(\mathrm{pACT}: L E C 2)^{40}$. The p35S:NLP7 construct was obtained by subcloning into $\mathrm{pBS}^{7}$. Protoplasts were co-transfected with the p35S:GUS plasmid (pRTL2-GUS (ref. 41)) as control. Eighteen hours after transfection, $\beta$-glucuronidase and luciferase activity were measured as described previously ${ }^{42,43}$. In short, protoplasts were extracted with a Triton X-100 containing buffer, and enzyme activities were measured using a fluorogenic or a light emitting assays with 4-methyl umbelliferyl glucuronide and luciferin, respectively, as substrate. Luciferase activity was expressed relative to p35S:GUS activity.

\section{References}

1. Lawler, D., Lemaire, G. \& Gastal, F. Plant Nitrogen: nitrogen, plant growth and crop yield. (eds Lea, P. \& Morot-Gaudry, J. F.) 343-368 (Springer, 2001).

2. Crawford, M. N. Nitrate: nutrient and signal for plant growth. Plant Cell 7, 859-868 (1995)

3. Wang, R., Okamoto, M., Xing, X. \& Crawford, N. M. Microarray analysis of the nitrate response in Arabidopsis roots and shoots reveals over 1,000 rapidly responding genes and new linkages to glucose, trehalose-6-phosphate, iron, and sulfate metabolism. Plant Physiol. 132, 556-567 (2003).

4. Scheible, W. R. et al. Genome-wide reprogramming of primary and secondary metabolism, protein synthesis, cellular growth processes, and the regulatory infrastructure of Arabidopsis in response to nitrogen. Plant Physiol. 136, 2483-2499 (2004).

5. Gutierrez, R. A. Systems biology for enhanced plant nitrogen nutrition. Science 336, 1673-1675 (2012).

6. Zhang, H. \& Forde, B. G. An Arabidopsis MADS box gene that controls nutrient-induced changes in root architecture. Science 279, 407-409 (1998).

7. Castaings, L. et al. The nodule inception-like protein 7 modulates nitrate sensing and metabolism in Arabidopsis. Plant J. 57, 426-435 (2009).

8. Rubin, G., Tohge, T., Matsuda, F., Saito, K. \& Scheible, W. R. Members of the LBD family of transcription factors repress anthocyanin synthesis and affect additional nitrogen responses in Arabidopsis. Plant Cell 21, 3567-3584 (2009).

9. Krouk, G., Mirowski, P., LeCun, Y., Shasha, D. E. \& Coruzzi, G. M. Predictive network modeling of the high-resolution dynamic plant transcriptome in response to nitrate. Genome Biol. 11, R123 (2010).

10. Schauser, L., Wieloch, W. \& Stougaard, J. Evolution of NIN-like proteins in Arabidopsis, rice, and Lotus japonicus. J. Mol. Evol. 60, 229-237 (2005).

11. Fornerod, M., Ohno, M., Yoshida, M. \& Mattaj, I. W. CRM1 is an export receptor for leucine-rich nuclear export signals. Cell 90, 1051-1060 (1997)

12. Fukuda, M. et al. CRM1 is responsible for intracellular transport mediated by the nuclear export signal. Nature 390, 308-311 (1997).

13. Haasen, D., Köhler, C., Neuhaus, G. \& Merkle, T. Nuclear export of proteins in plants: AtXPO1 is the export receptor for leucine-rich nuclear export signals in Arabidopsis thaliana. Plant J. 20, 695-705 (1999).

14. Roudier, F. et al. Integrative epigenomic mapping defines four main chromatin states in Arabidopsis. EMBO J. 18, 1928-1338 (2011).

15. Hu, H., Wang, Y. \& Tsay, Y. F. AtCIPK8, a CBL-interacting protein kinase, regulates the low-affinity phase of the primary nitrate response. Plant J. 57, 264-278 (2009).

16. Wang, R., Xing, X., Wang, Y., Tran, A. \& Craford, N. M. A genetic screen for nitrate regulatory mutants captures the nitrate transporter gene NRT1.1. Plant Physiol. 151, 472-478 (2009).

17. Ho, S. H., Lin, S. H., Hu, H. C. \& Tsay, Y. F. CHL1 functions as a nitrate sensor in plants. Cell 18, 1184-1194 (2009).

18. Konishi, M. \& Yanagisawa, S. Identification of a nitrate-responsive cis-element in the Arabidopsis NIR1 promoter defines the presence of multiple cisregulatory elements for nitrogen response. Plant J. 63, 269-282 (2010).

19. Thomas-Chollier, M., Herrmann, C., Defrance, M., Sand, O., Thieffry, D. \& van Helden, J. RSAT peak-motifs: motif analysis in full-size ChIP-seq datasets. Nucleic Acids Res. 40, e31 (2012).

20. Konishi, M. \& Yanagisawa, S. The regulatory region controlling the nitrateresponsive expression of a nitrate reductase gene, NIA1, in Arabidopsis. Plant Cell Physiol. 52, 824-836 (2011).

21. Girin, T. et al. Identification of a $150 \mathrm{bp}$ cis-acting element of the AtNRT2.1 promoter involved in the regulation of gene expression by the $\mathrm{N}$ and $\mathrm{C}$ status of the plant. Plant Cell Environ. 30, 1366-1380 (2007).
22. Blauwkamp, T. A., Chang, M. V. \& Cadigan, K. M. Novel TCF-binding sites specify transcriptional repression by Wnt signalling. EMBO J. 27, 1436-1346 (2008).

23. MacDonald, B. T., Tamai, K. \& He, X. Wnt/beta-catenin signaling: components, mechanisms, and diseases. Dev. Cell. 17, 9-26 (2009).

24. MacQuarrie, K. L., Fong, A. P., Morse, R. H. \& Tapscott, S. J. Genome-wide transcription factor binding: beyond direct target regulation. Trends Genet. 27, 141-148 (2011).

25. Meier, I. \& Somers, D. E. Regulation of nucleocytoplasmic trafficking in plants. Curr. Opin. Plant Biol. 14, 538-546 (2011).

26. Carmago, A. et al. Nitrate signaling by the regulatory gene NIT2 in Chlamydomonas. Plant Cell 19, 3491-3503 (2007).

27. Murthi, A. et al. Regulation of tRNA bidirectional nuclear-cytoplasmic trafficking in Saccharomyces cerevisiae. Mol. Biol. Cell. 21, 639-649 (2010).

28. Pierce, J. B., Eswara, M. B. \& Mangroo, D. The ins and outs of nuclear re-export of retrogradely transported tRNAs in Saccharomyces cerevisiae. Nucleus 1, 224-230 (2010).

29. Johnstone, A. D., Mullen, R. T. \& Mangroo, D. Plants, like mammals, but unlike Saccharomyces, do not regulate nuclear-cytoplasmic tRNA trafficking in response to nutrient stress. Plant Signal Behav. 6, 1183-1183 (2011).

30. Bernreiter, A. et al. Nuclear export of the transcription factor NirA is a regulatory checkpoint for nitrate induction in Aspergillus nidulans. Mol. Cell Biol. 27, 791-802 (2007).

31. Meur, G. et al. Nucleo-cytosolic shuttling of FoxO1 directly regulates mouse Ins2 but not Ins1 gene expression in pancreatic beta cells (MIN6). J. Biol. Chem 286, 13647-13656 (2011).

32. Gendrel, A. V., Lippmann, Z., Martienssen, R. \& Colot, V. Profiling histone modification patterns in plants using genomic tiling microarrays. Nat. Methods 2, 213-218 (2005).

33. Seifert, M., Gohr, A., Strickert, M. \& Grosse, I. Parsimonious higher-order hidden Markov models for improved array-CGH analysis with applications to Arabidopsis thaliana. PLoS Comput. Biol 8, e1002286 (2012).

34. Crowe, M. L. et al. CATMA: a complete Arabidopsis GST database. Nucleic Acids Res. 31, 156-158 (2003).

35. Lurin, C. et al. Genome-wide analysis of Arabidopsis pentatricopeptide repeat proteins reveals their essential role in organelle biogenesis. Plant Cell 16, 2089-2103 (2004).

36. Ge, Y., Dudoit, S. \& Speed, T. P. Resampling-based Multiple Testing for MicroarrayData Analysis. Test 12, 1 (2003).

37. Gagnot, S. et al. CATdb: a public access to Arabidopsis transcriptome data from the URGV-CATMA platform. Nucleic Acids Res. 36, D986-D990 (2008).

38. Danie l-Vedele, F. \& Caboche, M. A tobacco cDNA clone encoding a GATA-1 zinc finger protein homologous to regulators of nitrogen metabolism in fungi. Mol. Gen. Genet. 240, 365-373 (1993).

39. Thomine, S., Lelièvre, F., Debarbieux, E., Schroeder, J. I. \& Barbier-Brygoo, H. AtNRAMP3, a multispecific vacuolar metal transporter involved in plant responses to iron deficiency. Plant J. 34, 685-695 (2003).

40. Thévenin, J. et al. A new system for fast and quantitative analysis of heterologous gene expression in plants. New Phytol. 193, 504-512 (2012).

41. Carrington, J. C., Freed, D. D. \& Leinicke, A. J. Bipartite signal sequence mediates nuclear translocation of the plant potyviral NIa protein. Plant Cell $\mathbf{3}$, 953-962 (1991).

42. Jefferson, R. A., Kavanagh, T. A. \& Bevan, M. W. GUS fusions: betaglucuronidase as a sensitive and versatile gene fusion marker in higher plants. EMBO J. 6, 3901-3907 (1987).

43. Bailey-Serres, J. \& Dawe, R. K. Both $5^{\prime}$ and $3^{\prime}$ sequences of maize adh $1 \mathrm{mRNA}$ are required for enhanced translation under low-oxygen conditions. Plant Physiol. 112, 685-695 (1996).

\section{Acknowledgements}

This work was partly supported by an INRA/FAPESP grant to C.M. Work in the laboratory of V.C. is supported by the CNRS and the network of Excellence EPIGENESYS. We thank M. Lepetit and B. Dubreucq for the pNRT2.1:GUS and pACT:LEC2 plasmids, respectively, I. Debeaujon for Arabidopsis cell cultures, A. Bortolini Silveira for advice on ChIP, E. Biot and F. Granier for advice on data analysis, J. Strauss, M. Vincentz, J.P. Renou and the AReNA team for discussion, F. Daniel-Vedele for excellent comments on the manuscript, and A. Mallory for improving the English language.

\section{Author contributions}

C.Ma., F.R., L.C., C.M. and A.K. designed the experiments. C.Ma. performed most of the experiments and analysed the data. F.R. analysed ChIP-chip experiments. V.B. prepared samples for transcriptome experiments, E.B. performed and analysed CATMA hybridizations. C.Ma., F.R., V.C., C.M. and A.K. interpreted the results and wrote the manuscript. All authors discussed the results and the manuscript. 


\section{Additional information}

Supplementary Information accompanies this paper at http://www.nature.com/ naturecommunications

Competing financial interests: The authors declare no competing financial interests.
Reprints and permission information is available online at http://npg.nature.com/ reprintsandpermissions/

How to cite this article: Marchive C. et al. Nuclear retention of the transcription factor NLP7 orchestrates the early response to nitrate in plants. Nat. Commun. 4:1713 doi: $10.1038 /$ ncomms2650 (2013). 